\title{
Realistic single-neuron modeling
}

\section{William W. Lytton and John C. Wathey}

Realistic single-neuron modeling organizes and clarifies physiological hypotheses. It extends the experimenter's intuition and leads to testable predictions. A powerful new algorithm, several user-friendly software packages and the advent of fast, cheap computers have together made this tool accessible to a broad range of neurobiologists. Equally dramatic advances in experimental findings have increased the level of sophistication of the models. Here we provide a guide to singleneuron modeling, illustrate its power with a few examples and speculate on possible future directions for this rapidly growing field.

Key words: computer model/ compartmental model/ cable model/channel kinetics

ALL NEUROPHYSIOLOGISTS are modelers. They may explain the bursting behavior of a neuron in terms of voltage-sensitive calcium currents and a calcium-activated potassium conductance. They may conclude from a complex spike waveform that the cell has excitable dendrites. They may choose to voltage-clamp the cell body to study synaptic currents. Behind each of their experimental designs and interpretations of results lies an implicit hypothesis, a model, of how the neuron works.

Here we describe a tool with which these mental models can be transformed into precise and explicit computer simulations. This transformation is in itself a valuable exercise, because it requires a complete list of the relevant biophysical parameters. Compiling this list may immediately reveal important gaps in knowledge. Of far greater value, however, is the expansion of intuition that comes with running many simulations over wide ranges of parameter values.

Realistic models at the single-neuron level are primarily concerned with local changes in membrane current and voltage, caused by such things as synaptic input and voltage-sensitive conductances, and with the propagation of these local changes over spatially extensive dendrites and axons. With recent

From the Computational Neurobiology Laboratory, Salk Institute, 10010 N Torrey Pines Rd, La Jolla, CA 92037, USA (C) 1992 Academic Press Ltd

$1044-5765 / 92 / 010015+11 \$ 5.00 / 0$ advances, physiologists can now use modeling techniques previously accessible only to those with mathematical expertise and large computers.

What follows is not a comprehensive review of the subject but rather an overview for the uninitiated. We begin with some intuitive explanations of the biophysical processes being simulated and a guide to the latest simulation software. We then describe a few illustrative models, taken largely from our own work, and conclude with speculations on the future directions of this rapidly growing field.

\section{A guide to realistic single-neuron models}

\section{Modeling membrane mechanisms}

Biophysical processes that cause local changes in membrane potential, such as synaptic activation and voltage sensitive conductances, are most easily modeled in a patch of membrane that is spatially isopotential. This is an appropriate model for preparations such as the space-clamped squid axon ${ }^{1}$ or neurons that lack dendrites. ${ }^{2}$ Such models are best understood in terms of a parallel conductance circuit (Figure 1). The capacitor represents the electrical capacitance of the membrane, the batteries represent the reversal potentials of the various ionic channels in the membrane and the resistors symbolize the conductances of those channels. Those with arrows through them can vary over time. Probably the single most useful concept for understanding these models is that the membrane potential is given approximately by a weighted average of the various reversal potentials where the weighting factors are the corresponding ionic conductances. If one conductance, say $\mathrm{g}_{\mathrm{Na}}$, becomes much larger than all the others, then the membrane potential will be driven towards $\mathrm{E}_{\mathrm{Na}}$.

The time course of a synaptic conductance change depends in a complex way on the rates of diffusion and removal of neurotransmitter molecules in the synaptic cleft and on the kinetics of the reactions between transmitter and receptor molecules. Although these processes have been modeled in great detail, $, 3,4$ most models at the single-neuron level ignore the 


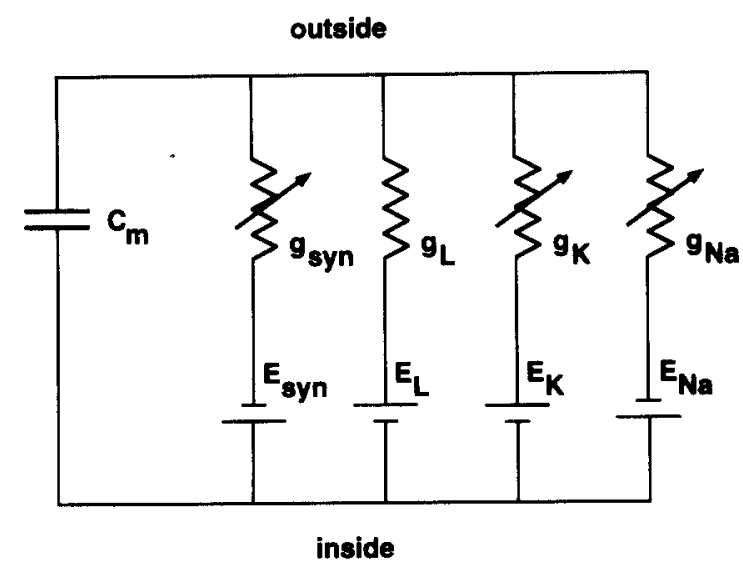

Figure 1. Parallel conductance model of active membrane with synapse. $\mathrm{C}_{\mathrm{m}}=$ membrane conductance; $\mathrm{I}=$ conductances of various channels; $\mathrm{E}=$ reversal potentials of various channels; syn = synapse; $L=$ nonspecific leakage; $\mathrm{K}=$ potassium channel; $\mathrm{Na}=$ sodium channel. (see text for explanation).

underlying complexity and use simple functions of time to approximate synaptic conductance waveforms. Examples are the alpha function ${ }^{5}$ and the product of exponentials (J.M. Bekkers and C.F. Stevens, personal communication). In a particularly interesting case the synaptic conductance depends on voltage as well as time. ${ }^{6}$

Most models of voltage-sensitive conductances follow the formalism introduced by Hodgkin and Huxley. 1 The essential concept is that of a microscopic 'gate' which covers the ion channel and makes all-or-none transitions between the open and closed states:

$$
\underset{\beta}{\text { closed }} \stackrel{\alpha}{\rightleftharpoons} \text { open }
$$

where the transition rates $\alpha$ and $\beta$ are functions of temperature and membrane potential. Their voltage dependence underlies the voltage sensitivity of the model conductance. The individual channels and their gates are not explicitly simulated; rather the model deals with a 'gating variable' that describes the average behavior of a large population of gates of a given channel type. The gating variable specifies the fraction of gates in the open state at any instant in time. There is no simple expression for the gating variable; its value can only be approximated by numerical integration of the differential equations that arise from the underlying all-or-none transition behavior and from the rate functions $\alpha$ and $\beta$. A detailed explanation of these differential equations

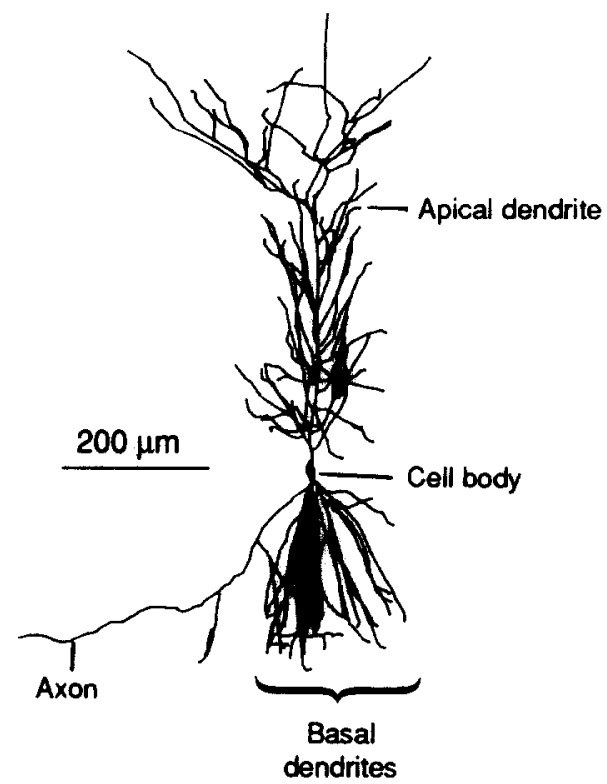

Figure 2. Computer-generated drawing of a hippocampal CA1 pyramidal neuron. The cell was filled in vitro with horseradish peroxidase and digitized from a wholemount of the slice (data courtesy D. Amaral). The axon is the long process at lower left. The cell has four basal dendrites (below the cell body) and one apical dendrite. Together they comprise 93 branches totaling $14 \mathrm{~mm}$ in length. Reproduced from ref 11 , with permission.

and of the derivation of the rate functions from voltage-clamp data are given in ref 7 .

\section{Modeling cable properties}

A neuron with extensive dendrites (Figure 2) cannot be adequately modeled as a spatially isopotential patch of membrane. In such a cell a local change in membrane potential propagates across the cell over time. This propagation is described by a partial differential equation known as the cable equation. ${ }^{8}$ It is mathematically identical to the diffusion equation, and its behavior is perhaps most easily understood by analogy to diffusion. Imagine a long thin tube filled with water. At time zero a fixed amount of a soluble dye is injected into the tube somewhere along its length. At that instant the concentration of the dye is zero over most of the length of the tube but is high near the injection site (Figure 3). The diffusion equation states that the rate of change of concentration of the dye over time is proportional to the curvature of the concentration profile over distance. The effect is that local bumps in the concentration profile tend to smooth out over time. If the tube in 
our example is itself slightly permeable to the dye, then the dye will not only diffuse down the length of the tube but some of it will also diffuse across the walls of the tube and be lost to the surrounding medium. This case is directly analogous to the spatially extensive neuron: the tube is the neuronal membrane, the dye is electric current and its local concentration is the membrane potential.

For most realistic models, numerical integration must be used to approximate the solution to the cable equation. Because it is a partial differential equation in both space and time, its numerical solution requires not only dividing time into discrete steps but also dividing the neuron into discrete spatial increments (commonly called compartments or segments). Each of these is considered isopotential and may have associated with it various membrane mechanisms (e.g. voltage-sensitive conductances) that can affect the membrane potential at that location. Such a model therefore requires the numerical integration of a large system of coupled differential equations: the cable equation and the various ordinary differential equations describing local membrane mechanisms in the compartments. These equations do not yield easily to numerical integration. When conventional methods are used, the solutions become unstable with large time steps or with small compartment sizes, ${ }^{9}$ but a powerful new algorithm ${ }^{10}$ has solved the stability problem for models of highly branched neurons, a breakthrough that has opened up a new realm of neuronal modeling. Problems that once required hours or days of computer time can now be solved in seconds. ${ }^{11-15}$

\section{Software}

There are several important criteria for judging neuronal simulation software. The most important is the reliability of the underlying design. Since the main task of these programs is numerical integration, a critical test of their correctness is that their results converge to analytical solutions in those simplified cases for which analytical solutions are known. A neuronal simulator must also be flexible. Most programs achieve this through the use of a high-level interpreted language that controls the simulation. The best are distributed with sample interpreter scripts that show by example how their features are to be used. Simulations of morphologically realistic neurons require a sophisticated algorithm ${ }^{10}$ and some means of reading digitized morphological data. A simulator should also display results graphically in a way that simplifies interpretation.

Several software packages meet most or all of these criteria. We use the programs of Michael Hines, ${ }^{16}$ the latest of which is NEURON. A unique and powerful feature of NEURON is its model description language, a high-level language with which sophisticated local membrane mechanisms can easily be created. The Genesis simulator ${ }^{17}$ is relatively easy to use and features a sophisticated graphical display. Other popular packages include Nemosys, ${ }^{15}$ SPICE ${ }^{18}$ and Saber. ${ }^{19}$ Most of these run only on workstations but there is a $\mathrm{PC}$ version of NEURON.

\section{Examples}

\section{Passive cable properties}

A criticism often raised against realistic neuronal modeling is that many of the requisite parameters are unknown, so modeling efforts are premature. We would argue that the goal of modeling is not to confirm the completeness of physiological knowledge but instead to contribute to that knowledge by identifying gaps within it and by aiding in the interpretation of data.

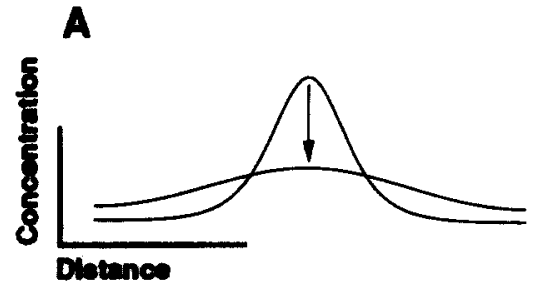

B

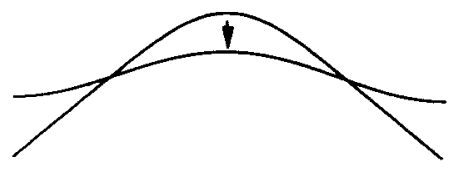

Figure 3. Diffusion along a sealed tube. (A) A large concentration difference between the center and its surroundings (high spatial curvature) leads to a rapid drop in concentration at the center (arrow). (B) A more gentle initial curvature leads to a slower drop in concentration. 
A good example is the controversy over one of the fundamental parameters of passive cable models, the specific membrane resistance $R_{m}$. It is not directly measurable but must be inferred, using a cable model, from measurements of cellular input resistance. The problem is that these measurements may differ by a factor of 10 depending on whether impaling or tight-seal electrodes are used..$^{20,21}$ This uncertainty in $R_{m}$ has drastic effects on the behavior of single-neuron models and on the interpretation of experimental results. ${ }^{22}$ Figure 4 shows a simple example, the effect of $R_{m}$ on steady-state depolarization across a dendritic tree. The discrepancy in measurements of input resistance has been attributed to current leak around the impaling electrode, cellular dialysis by the tight-seal electrode, damage to distal dendrites in some preparations ${ }^{23}$ and variation in the age of the animals in others (J.F. Storm, personal communication). A recent modeling study has shown that differences in background synaptic activity can also have large effects on input resistance. ${ }^{14}$ Spruston and Johnston ${ }^{24}$ recently measured input resistances between the two extremes using a perforated-patch technique, which avoids some of the artifacts of impalement and conventional tight-seal recording. They successfully used cable models, both analytic and numerical, to interpret discrepancies between their results and those obtained with impaling electrodes. Their models of leakage around the impaling electrode quantitatively predicted the lower input resistance and time constants measured with that technique.

Simple models can often aid the interpretation of experimental results and can be readily performed by the experimenter. In a study of synaptic connectivity in an isolated leech ganglion, ${ }^{25}$ a dendrite of an identified, Lucifer-yellow-filled neuron was ablated by irradiation. The ablation caused a reduction in an inhibitory postsynaptic potential (i.p.s.p.) from a particular inhibitory input, suggesting that the lesioned dendrite was the location of the input. The irradiation also, however, caused damage to the cell body resulting in a large reduction in input impedance, which would be expected to reduce the size of postsynaptic potentials. Was the observed reduction in the i.p.s.p. caused by the dendritic ablation or by the general damage to the cell body? A five-compartment passive model was used to assess

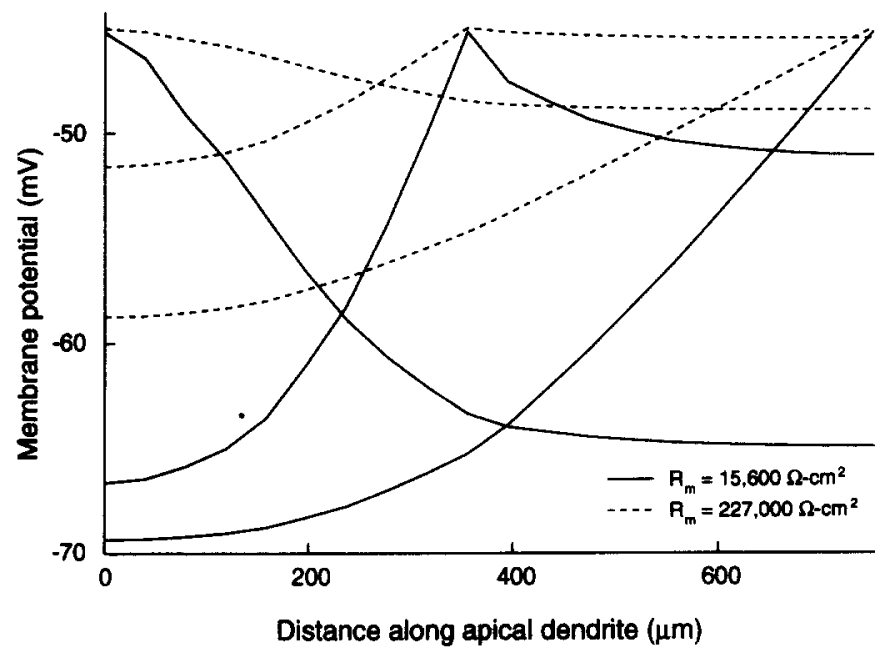

Figure 4. Steady-state membrane potential responses to continuous current injection into the cell body or various sites along the primary apical dendrite of the cell shown in Figure 2. The entire cell was passive and the resting potential was $-70 \mathrm{mV}$. Each curve represents a separate simulation run. The maximum of each curve indicates the location of the current injection. Solid and dashed lines show results from simulations using membrane resistances of $15,600 \Omega \mathrm{cm}^{-2}$ and $227,000 \Omega \mathrm{cm}^{-2}$, respectively. The corresponding input resistances were 50 and $500 \mathrm{M \Omega}$, respectively. The amount of current was adjusted to compensate for differences in input resistance and varied from $0.5 \mathrm{nA}$ at the cell body to $0.063 \mathrm{nA}$ at the distal tip of the low-resistance cell. The corresponding range was 0.05 to $0.027 \mathrm{nA}$ for the high-resistance cell. Note the more rapid decay of potential with distance in the proximal half of the dendrite, where the density of secondary dendritic branches is greatest. Reproduced from ref 11 , with permission. 

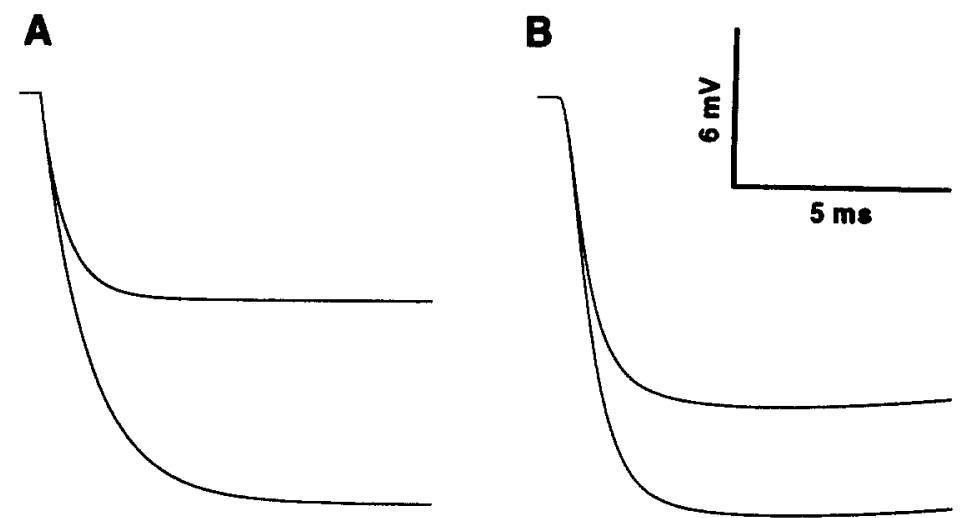

Figure 5. Changes in synaptic response do not always parallel changes in input impedance. (Simulations of experimental results from ref 25.) (A) Injection of $0.5 \mathrm{nA}$ of current produces $50 \%$ less hyperpolarization after changing the membrane resistance of the cell body. This implies a $50 \%$ reduction in input resistance as measured at the cell body. (B) The same change in cell body membrane resistance reduces the effect of a distal inhibitory synaptic input by only $25 \%$.

the effect of the change in membrane resistance on the i.p.s.p. size. The model was altered to match the input impedance before and after irradiation and to match the i.p.s.p. size in the control. Interestingly, the change in membrane resistance had much less effect on the i.p.s.p. than on an injected current (Figure 5), because the i.p.s.p. was itself so large that it already enormously reduced membrane resistance, making the reduction due to cell damage less significant. The reduction in the i.p.s.p. seen in the experiment therefore appeared to be a specific effect of dendritic ablation.

\section{Relating voltage- and current-clamp data}

A model can be used to evaluate the artifacts intrinsic to voltage-clamp studies: deficiencies in space clamp, difficulties with rapidly sourcing or sinking currents, variable temperature dependence and damage to dissociated cells. Ideally a model derived from voltage-clamp data should be able to reproduce the behavior of the current-clamped neuron.

A modeling study of this sort has been used to correlate voltage-clamp studies of the T-type calcium channel in thalamic neurons with the low-threshold spike that depends on it. ${ }^{26}$ There have been three voltage-clamp studies of this channel in thalamic cells; ${ }^{27-29}$ although they are in overall agreement as to the approximate range of activation of the channel, the exact parameters vary considerably (Figure 6A).
A study using cell impalement ${ }^{29}$ produced a steep steady-state activation $\left(m_{\infty}\right)$ curve, whereas studies using whole-cell patch produced shallower curves. Our modeling revealed that a low-threshold calcium spike could be more readily produced using the steeper curve (Figure 6B). A low-threshold calcium spike could be obtained using the shallower curve (Figure 6C) if a large persistent sodium current was used to offset the effect of a less steep activation of the $T$ current. The low-threshold calcium spike produced with these parameters was far more sensitive to the exact amount of hyperpolarization employed to elicit it. Since both types of voltageclamp recording are subject to artifacts, this type of modeling can provide a valuable clue for interpreting the results from the two techniques.

Using the same approach, the effect of the drug ethosuximide on the $T$ channel was assessed. Our modeling of the voltage-clamp traces from a recent study of the action of ethosuximide on isolated thalamic cells showed that the effect of the drug was to change the voltage dependence of the $T$ channel activation curve rather than to simply reduce the overall conductance. ${ }^{30}$ Further modeling of currentclamp data suggested that this alteration in channel behavior would be able to prevent or reduce low-threshold calcium spikes and thereby alter the behavior of the cell in the thalamic circuit, as had been previously suggested. As ethosuximide is effective in treating absence epilepsy, a particular type of epilepsy dependent on thalamocortical interaction ${ }^{31}$, this result supports 
A $m_{0}$

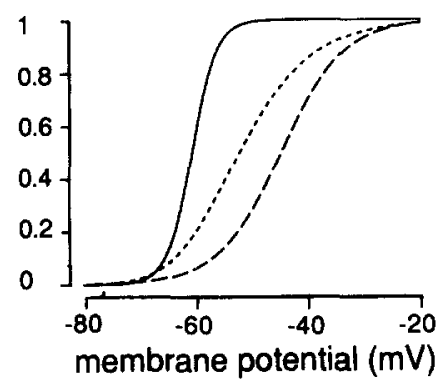

B
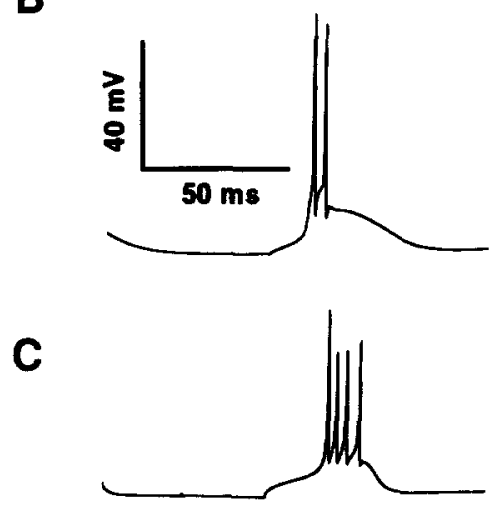

Figure 6. Voltage clamp results differ among studies using different techniques. (A) Steady-state activation $\left(m_{\infty}\right)$ curves for the $\mathrm{T}$ calcium channel as reported in three voltage-clamp studies: solid line $;{ }^{29}$ short dashes; ${ }^{28}$ and long dashes. ${ }^{27}$ All show a threshold at about $-70 \mathrm{mV}$ but they have different slopes. (B) Low-threshold calcium spike obtained after hyperpolarization in a simulation using the solid-line $m_{\infty}$ curve in (A). This spike is robust: it occurs following various degrees of hyperpolarization. (C). Less robust low-threshold calcium spike obtained after hyperpolarization in a simulation using the middle $m_{\infty}$ curve in (A). It is difficult to find parameters that give spiking behavior and the spike occurs only for a narrow range of hyperpolarization. (W. Lytton, unpublished simulations).

the hypothesis that the drug might exert its antiepileptic activity through its action on the $T$ channel. ${ }^{30}$ Because models are easier to manipulate than experimental preparations, models could potentially be used to predict not only the effect of a particular channel modification on cell firing patterns but also channel modifications that might abolish a pathological firing pattern.

\section{Changes in dendritic excitability}

Our last example concerns an enigmatic aspect of synaptic plasticity in the hippocampus. Hippocampal pyramidal neurons show a robust form of synaptic plasticity termed long-term potentiation (LTP), in which a brief, high-frequency stimulus to the afferent fibers causes an enhanced response to subsequent single test stimuli. This enhancement is usually measured as increases in the amplitude (or initial slope) of the excitatory postsynaptic potential (e.p.s.p.) and in the probability of firing of the postsynaptic neurons (evidenced by an increase in the size of the extracellular population spike). An important characteristic of LTP in the CA1 region of the hippocampus is that it is specific to those synapses that received the tetanic (high frequency) stimulation. ${ }^{32,33}$

One puzzling aspect of the LTP story has, however, received relatively little attention. In many LTP experiments there is a greater increase in the probability of firing than can be accounted for by the potentiation of the e.p.s.p. This is most easily seen in the curve relating population spike size to e.p.s.p. slope. Typically the effect of LTP is to shift this curve to the left, which indicates that an e.p.s.p. of a given slope is more likely to fire the cell after LTP than before (Figure 7). The phenomenon is also evident in some intracellular recordings ${ }^{34}$ in which the probability of firing increases after the tetanus, although the size and shape of the e.p.s.p. does not change (Figure 8A,B). This component of LTP is called potentiation of e.p.s.p.-to-spike coupling, or simply E-S potentiation. ${ }^{33,35}$

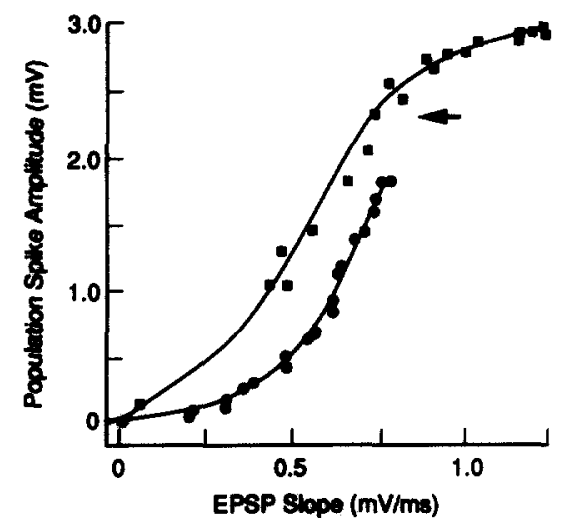

Figure 7. High-frequency stimulation causes a leftward shift in the plot of population spike size versus e.p.s.p. slope. One possible explanation is that the conditioning stimulus causes a long-term increase in dendritic excitability. Unpublished data courtesy L. Chavez-Noriega and T. Bliss. 

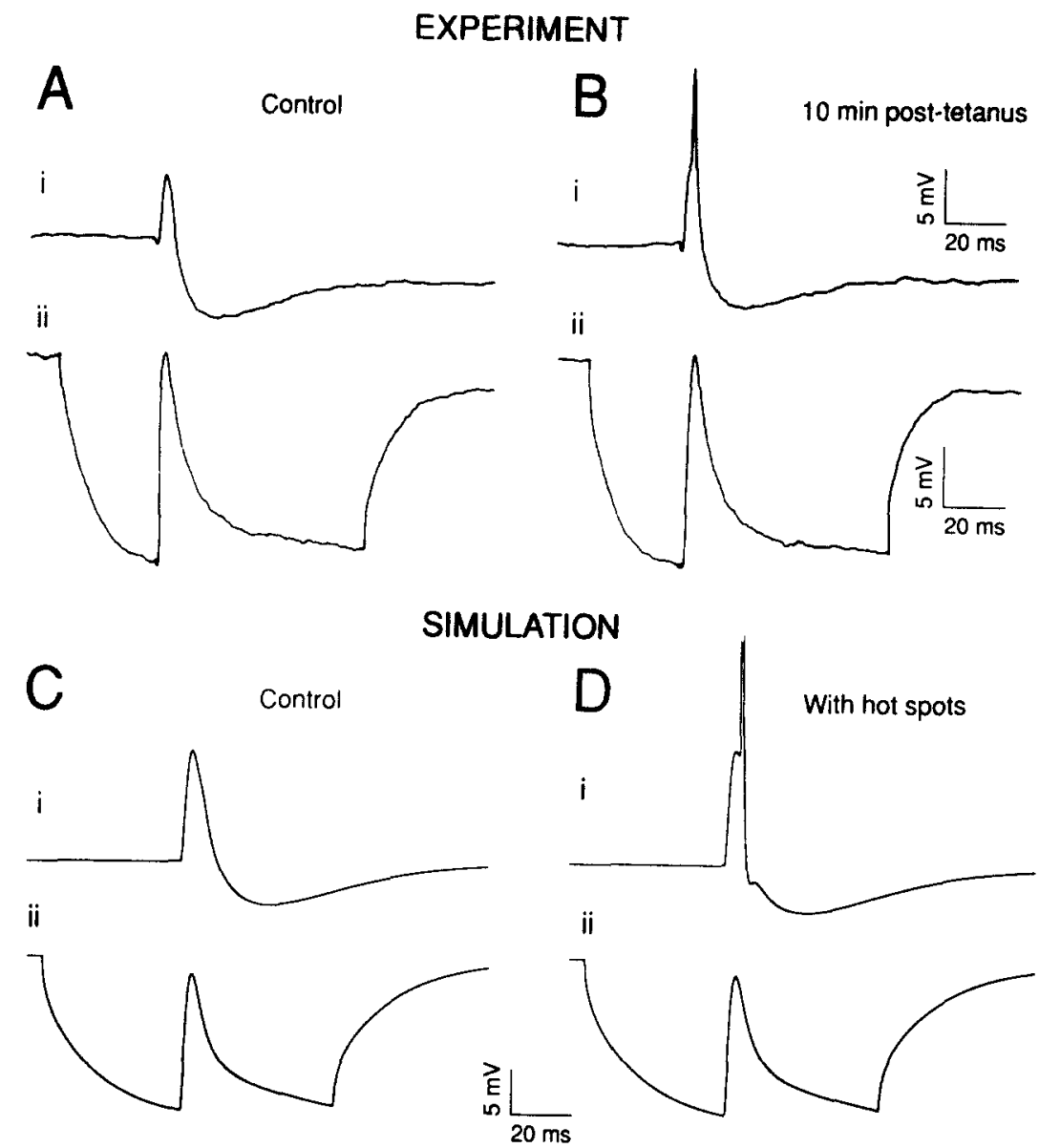

Figure 8. (A),(B). Intracellular evidence for E-S potentiation. (A)i. A test shock to afferent fibers elicits a subthreshold response in an intradendritic recording from a hippocampal CA1 pyramidal cell. In (B)i, following tetanization of this synaptic input, the response to the same test shock is suprathreshold. (A)ii and (B)ii shows that the e.p.s.p. is not significantly altered by the tetanus. From ref 11 (after ref 34), reproduced with permission. (C),(D) Simulation of an intradendritic recording showing E-S potentiation. (C)i. A test shock which excites 18 excitatory and 14 inhibitory synaptic contacts on the apical dendrite produces a subthreshold e.p.s.p. (D)i. Testing the assumption that tetanic stimulation increases dendritic excitability by adding voltage-sensitive calcium conductance (hot spots) near the tetanized synaptic contacts. This results in E-S potentiation: the test stimulus has become suprathreshold. (C)ii and (D)ii The response embedded in a $0.3 \mathrm{nA}$ hyperpolarizing current pulse shows that the e.p.s.p. is unchanged. Reproduced from ref 11, with permission.

We used a model to investigate some of the consequences of the hypothesis that E-S potentiation is caused by an increase in postsynaptic excitability. ${ }^{11}$ The cellular morphology used in these simulations was identical to that in Figure 2. The axon and soma were excitable but the dendrites were initially passive. We assumed that the effect of the tetanus was to unmask voltage-sensitive calcium channels near the sites of the tetanized synaptic contacts. In the model this involved adding a 'hot spot' of voltage-sensitive calcium permeability to those dendritic compartments receiving excitatory synaptic input. This local change in dendritic excitability mimicked the intracellular manifestations of E-S potentiation (Figure 8C,D).

The more important issue, however, is the effect of this change in excitability on the specificity of LTP. To quantify the specificity of the simulated E-S potentiation, we ran a series of simulations in which the hot spots were colocalized with a subset $(40 / 80)$ 
of synaptic contacts, denoted tetanized. We then compared the effects of the hot spots on these and on the remaining (untetanized) synapses. Both groups of synapses were scattered randomly over restricted regions of the dendrites. The strength of the hot spots (which can be thought of as the number of calcium channels they comprise) was in every case adjusted to reduce the firing threshold of the cell by $10 \%$ through the tetanized contacts. If the hot spots had a negligible effect on the threshold for firing the cell through the untetanized contacts, then the simulated E-S potentiation was specific to the tetanized synapses. If both groups of synapses were affected, then the E-S potentiation was nonspecific.

We defined specificity as the difference between the changes in threshold at the two synaptic groups divided by the sum of these quantities. With this definition, specificity is 1.0 if the untetanized synapses are unaffected by the hot spots; it is 0 if the two groups of synapses are equally affected and it approaches -1.0 if the hot spots have a greater effect on the untetanized than on the tetanized synapses. Much to our surprise, we found that the specificity could lie anywhere along this spectrum, depending on the spatial distribution of the synapses (Figure 9).

We ran many simulations with different patterns of synaptic input and, eventually, some trends emerged. Specificity tends to be high if the untetanized contacts are, on average, closer to the soma than the tetanized contacts (Figure 9A). This tendency persists even if the hot spots are not colocalized with the tetanized contacts but are instead confined to the most proximal $80 \mu \mathrm{m}$ of the five primary dendrites (Figure 9B). The highest specificities are obtained when the two groups of synapses are completely segregated to different primary dendrites (Figure 9C) but, even in this case, specificity approaches 0 if the untetanized contacts are sufficiently far from the cell body. We can understand these results by noting that it is difficult to fire the cell using synapses that are far from the spike initiating zone. The threshold synaptic strength required to fire the cell through distant inputs is therefore disproportionately sensitive to changes in dendritic excitability.

These results show that E-S potentiation by an increase in dendritic excitability can appear to be highly specific and cannot therefore be ruled out solely by the experimental observation that LTP is synapse-specific in CA1 pyramidal cells. The model also makes a prediction: if a significant component of E-S potentiation occurs by this mechanism, then
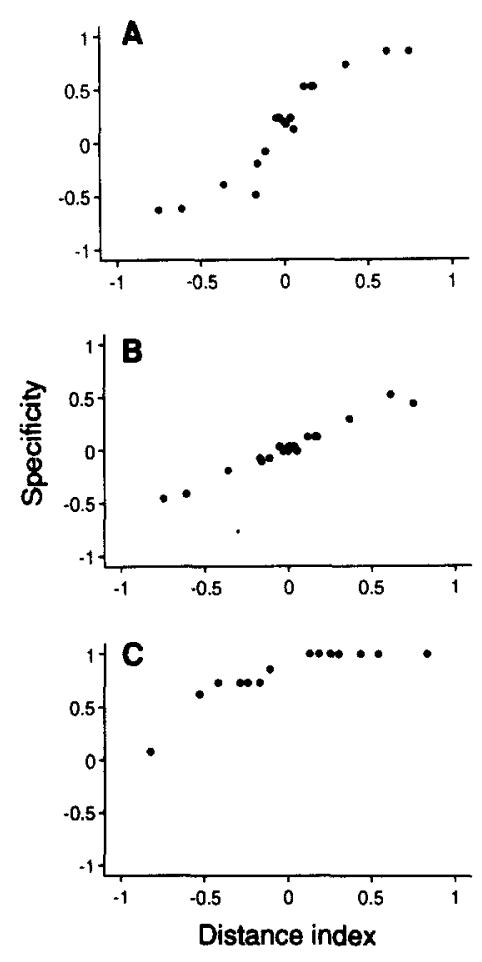

Figure 9. Simulations to test the specificity of E-S potentiation for different spatial distributions of synaptic input. (A) Forty tetanized and forty untetanized synaptic contacts were scattered at random on the apical dendrite. 'Hot spots' of voltage-sensitive $\mathrm{Ca}^{2}+$ conductance were added to those compartments receiving tetanized contacts, such that the threshold for firing the cell through the tetanized input was reduced by $10 \%$. Specificity (vertical axis) is a measure of the effect of these hot spots on the untetanized input. Positive values indicate a greater effect on the tetanized than on the untetanized input, negative values the reverse; 0 indicates equal effect on the two inputs. Distance index (horizontal axis) is a measure of the relative distances of the tetanized and untetanized inputs from the cell body, as judged by their effectiveness at firing the cell. Positive values indicate that the untetanized input is closer than the tetanized input, negative values the reverse; 0 indicates that the two inputs are equidistant from the cell body. E-S potentiation tends to be specific if the untetanized input is closer to the cell body than the tetanized input. (B) As (A), except that the hot spots were added to the most proximal $80 \mu \mathrm{m}$ of the five primary dendrites, rather than to the sites of the tetanized synaptic contacts. As in (A), specificity and distance index appear to be correlated. (C). As (A), except that the tetanized and untetanized inputs are segregated to apical and basal dendrites (see Figure 2) respectively. Hot spots were co-localized with tetanized contacts. Here E-S potentiation is highly specific over a wide range of distance index values. (Reproduced from ref 11, with permission). 
that component will appear nonspecific if the untetanized synapses are sufficiently far from the soma relative to the tetanized synapses.

In preliminary experiments of this kind (S. Chattarji, unpublished data; see also ref 36) tetanization of a proximal input to CA1 neurons causes potentiation of the population spikes elicited through both the tetanized input and a distal untetanized input, in agreement with the prediction. Recently evidence of a nonspecific component of LTP in CA1 neurons has also been reported (37) but it cannot be seen if the tetanized and untetanized inputs are segregated to basal and apical dendrites, respectively, consistent with the simulation results of Figure 9C.

This example illustrates several virtues of realistic modeling. The simulations suggest a way to reconcile seemingly contradictory experimental results (the evidence for postsynaptic excitability changes and the specificity of LTP). They reveal an important determinant of specificity (the relative distances of the two inputs from the soma). In so doing they suggest an experiment by which the effects of postsynaptic excitability changes might be better isolated from the other effects of tetanization. The model also has limitations. It ignores other possible mechanisms for E-S potentiation ${ }^{35}$ and omits much of the known physiological complexity of hippocampal pyramidal cells. ${ }^{38,39}$ The goal of the model was not, however, to reproduce the complete behavior of the neuron but was to give insight into some consequences of changes in dendritic excitability. In the future more complete models should provide better insight.

\section{Future directions}

In the near term we foresee a trend toward greater detail and realism in the models. One promising and largely unexplored direction is the modeling of voltage-sensitive conductances using reaction schemes and rate functions inferred from single-channel recordings. In many models, realism is now limited by the lack of data on the spatial distribution of voltage-sensitive channels across the cell. Many of these channels have now been characterized by molecular biological techniques ${ }^{40}$ and histochemical labeling is likely to play an increasingly important role in estimating channel densities. ${ }^{41}$ Imaging of dendritic current fluxes using ion-sensitive dyes will also contribute to this effort. ${ }^{42}$ We expect more highly constrained experiments done with the needs of modeling in mind. It might be possible, for example, to estimate the spatial distribution of dendritic $\mathrm{Ca}^{2}+$ channels from whole-cell voltageclamp data taken in the presence of a known and controllable spatial gradient of some channel blocker. Although the inadequacy of the space clamp would preclude any simple-minded interpretation of the results, a detailed model based on physiological and morphological data from the same neuron might provide a meaningful answer. Some work has already approached this level of sophistication. ${ }^{43}$ Another promising direction is the modeling of second-messenger systems that regulate ionic conductances ${ }^{44,45}$ and are involved in some forms of synaptic plasticity. ${ }^{46}$

Future models may address more profound questions concerning the computational roles of dendritic trees and neuronal dynamics. Models can already identify neurons that are likely to be capable of extensive dendritic computation ${ }^{47}$ and a few imaginative models have shown what such computation might achieve. ${ }^{22,48}$ The role of neuronal dynamics will probably be most easily understood in simplifying models that try to capture the essence of the behavior using the smallest possible number of state variables. ${ }^{49,50}$ This dimensional reduction approach may produce models that retain the computational power of biological neurons, yet are sufficiently simplified that they can be used in large network models. The seeds of this important future trend are already evident in the pioneering work of Traub and colleagues (this issue ${ }^{51}$ ). An important technological trend will be the implementation of these realistic network models on massively parallel computers. ${ }^{52}$.

Long-term potentiation in the hippocampus is treated in detail in Seminars in the Neurosciences (1990) volume 2, number 5, 'Associative Longterm Synaptic Chances' edited by B. Gustafsson and $\mathrm{H}$. Wigström.

\section{References}

1. Hodgkin AL, Huxley AF (1952) A quantitative description of membrane current and its application to conduction and excitation in nerve. J Physiol (Lond) 117:500-544

2. Yamada WM, Koch C, Adams PR (1989) Multiple channels and calcium dynamics, in Methods in Neuronal Modeling (Koch C, Segev I, eds), pp 97-133. MIT Press, Cambridge, MA 
3. Bartol TM, Land BR, Salpeter EE, Salpeter MM (1991) Monte Carlo simulation of miniature endplate current generation in the vertebrate neuromuscular junction. Biophys J 59:1290-1307

4. Wathey JC, Nass MM, Lester HA (1979) Numerical reconstruction of the quantal event at nicotinic synapses. Biophys J 27:145-164

5. Jack JJB, Noble D, Tsien RW (1983) Electric Current Flow in Excitable Cells. Oxford University Press, New York

6. Zador A, Koch C, Brown TH (1990) Biophysical model of a Hebbian synapse. Proc Natl Acad Sci USA 87:6718-6722

7. Cronin J (1987) Mathematical Aspects of Hodgkin-Huxley Neural Theory. Cambridge University Press, New York

8. Rall W (1989) Cable theory for dendritic neurons, in Methods in Neuronal Modeling (Koch C, Segev I, eds), pp 9-62. MIT Press, Cambridge, MA

9. Mascagni MV (1989) Numerical methods for neuronal modeling, in Methods in Neuronal Modeling (Koch C, Segev I, eds), pp 439-484. MIT Press, Cambridge, MA

10. Hines ML (1984) Efficient computation of branched nerve equations. Int $J$ Biomed Comput 15:69-76

11. Wathey JC, Lytton WW, Jester JM, Sejnowski TJ (1992) Computer simulations of EPSP-spike (E-S) potentiation in hippocampal CA1 pyramidal cells. J Neurosci, in press

12. Lytton WW, Sejnowski TJ (1991) Inhibitory interneurons may help synchronize oscillations in cortical pyramidal neurons. J Neurophysiol 66:1059-1079

13. Lytton WW (1991) Simulations of a phase comparing neuron of the electric fish Eigenmannia. J Comp Physiol A 169:117-125

14. Bernander O, Douglas RJ, Martin KAC, Koch C (1992) Synaptic background activity determines spatio-temporal integration in single pyramidal cells. Proc Natl Acad Sci $\mathrm{USA}$, in press

15. Miller JP (1990) Computer modelling at the single-neuron level. Nature 347:783-784

16. Hines ML (1989) A program for simulation of nerve equations with branching geometries. Int $\mathrm{J}$ Biomed Comput 24:55-68

17. Wilson MA, Bower JM (1989) The simulation of large-scale neural networks, in Methods in Neuronal Modeling (Koch C, Segev I, eds), pp 291-334. MIT Press, Cambridge, MA

18. Segev I, Fleshman JW, Burke RE (1989) Compartmental models of complex neurons, in Methods in Neuronal Modeling (Koch C, Segev I, eds), pp 291-334. MIT Press, Cambridge, MA

19. Carnevale NT, Woolf TB, Shepherd GM (1990) Neuron simulations with SABER. J Neurosci Meth 33:135-148

20. Brown TH, Fricke RA, Perkel DH (1981) Passive electrical constants in three classes of hippocampal neurons. J Neurophysiol 4:812-827

21. Edwards FA, Konnerth A, Sakmann B, Takahashi T (1989) A thin slice preparation for patch clamp recordings from neurones of the mammalian central nervous system. Pflügers Arch 41:600-612

22. Brown TH, Zador AM, Mainen ZF, Claiborne BJ (1992) Hebbian computations in hippocampal dendrites and spines, in Single Neuron Computation (McKenna T, Davis J, Zornetzer SF, eds). Academic Press, New York, in press

23. Kay AR, Wong RKS (1987) Calcium current activation kinetics in isolated pyramidal neurones of the CA1 region of the mature guinea-pig hippocampus. J Physiol (Lond) 392:603-616

24. Spruston N, Johnston D (1992) Perforated patch-clamp analysis of the passive membrane properties of three classes of hippocampal neurons. J Neurophysiol, in press
25. Lytton WW, Kristan WB (1989) Localization of a leech inhibitory synapse by photo-ablation of individual dendrites. Brain Res 504:43-48

26. Jahnsen H, Llinas R (1984) Ionic basis for the electroresponsiveness and oscillatory properties of guinea-pig thalamic neurons in vitro. J Physiol (Lond) 349:227-247

27. Hernandez-Gruz A, Pape HC (1989) Identification of two calcium currents in acutely dissociated neurons from the rat lateral geniculate nucleus. J Neurophysiol 61:1270-1283

28. Coulter DA, Huguenard JR, Prince DA (1989) Calcium currents in rat thalamocortical relay neurones: kinetic properties of the transient low-threshold current. J Physiol (Lond) 414:587-604

29. Crunelli V, Lightowler S, Pollard CE (1989) A T-type $\mathrm{Ca}^{2+}$ current underlies low-threshold $\mathrm{Ca}^{2+}$ potentials in cells of the cat and rat lateral geniculate nucleus. J Physiol (Lond) 413:543-561

30. Coulter DA, Huguenard JR, Prince DA (1989) Characterization of ethosuximide reduction of low-threshold calcium current in thalamic neurons. Ann Neurol 25:582-593

31. Williams D (1953) A study of thalamic and cortical rhythms in petit mal. Brain 76:50-69

32. Brown TH, Chapman PF, Kairiss EW, Keenan CL (1988) Long-term synaptic potentiation. Science 242:724-728

33. Gustafsson B, Wigström H (1990) Associative long-lasting modifications in synaptic efficacy. Semin Neurosci 2: no 5

34. Taube JS, Schwartzkroin PA (1988) Mechanisms of longterm potentiation: EPSP / spike dissociation, intradendritic recordings, and glutamate sensitivity. J Neurosci 8:1632-1644

35. Chavez-Noriega LE, Bliss TVP (1991) Persistent modulation of neural excitability in the hippocampus: the role of EPSP-spike (E-S) potentiation, in Glutamate, Cell Death and Memory (Asher P, Choi D, Christen P, eds), pp 37-44. Springer, Heidelberg

36. Wathey JC, Ghattarji S, Lytton WW, Jester JM, Sejnowski TJ (1991) Computer simulations of E-S potentiation in hippocampal CA1 pyramidal cells. Soc Neurosci Abstr 17:385

37. Hess G, Gustafsson B (1990) Changes in field excitatory postsynaptic potential shape induced by tetanization in the CA1 region of the guinea-pig hippocampal slice. Neuroscience 37:61-69

38. Llinas RR (1988) The intrinsic electrophysiological properties of mammalian neurons: insights into central nervous system function. Science 242:1654-1664

39. Traub RD, Wong RKS, Miles R, Michelson H (1991) A model of a CA3 hippocampal pyramidal neuron incorporating voltage-clamp data on intrinsic conductances. $J$ Neurophysiol 66:635-650

40. Catterall WA (1988) Structure and function of voltagesensitive ion channels. Science 242:50-61

41. Westenbroek RE, Ahlijanian MK, Catterall WA (1990) Clustering of $\mathrm{L}$-type $\mathrm{Ca}^{2+}$ channels at the base of major dendrites in hippocampal pyramidal neurons. Nature 347:281-284

42. Regehr WSG, Connor JA, Tank DW (1989) Optical imaging of calcium accumulation in hippocampal pyramidal cells during synaptic activation. Nature 341:533-536

43. Cauller LJ, Connors BW (1992) Functions of very distal dendrites: experimental and computational studies of layer I synapses on neocortical pyramidal cells, in Single Neuron Computation (McKenna T, Davis J, Zornetzer SF, eds), pp 199-229. Academic Press, New York, in press

44. Chay TR (1990) Effect of compartmentalized $\mathrm{Ca}^{2+}$ ions on electrical bursting activity of pancreatic beta-cells. Am J Physiol 258:C955-965 
45. Nicoll RA (1988) The coupling of neurotransmitter receptors to ion channels in the brain. Science 241:545-551

46. Gingrich KJ, Byrne JH (1987) Single-cell neuronal model for associative learning. J Neurophysiol 57:1705-1715

47. Bloomfield SA, Sherman SM (1989) Dendritic current flow in relay cells and interneurons of the cat's lateral geniculate nucleus. Proc Natl Acad Sci USA 86:3911-3914

48. Shepherd GM, Woolf TB, Carnevale NT (1989) Comparisons between active properties of distal dendritic branches and spines: implications for neuronal computations. J Cog Neurosci 1:273-286
49. Rose RM, Hindmarsh JL (1989) The assembly of ionic currents in a thalamic neuron: III The seven-dimensional model. Proc R Soc Lond B 237:313-334

50. FitzHugh R (1961) Impulses and phsyiological states in theoretical models of nerve membrane. Biophys J 1:445-466

51. Traub RD, Miles R (1992) Modeling hippocampal circuitry using data from whole cell patch clamp and dual intracellular recordings in vitro. Semin Neurosci 4:27-36

52. Mascagni $\mathbf{M}$ (1991) A parallelizing algorithm for computing solutions to arbitrarily branched cable neuron models. J Neurosci Meth 36:105-114 